\title{
Selective Recognition and Detection of L-Aspartic Acid by Molecularly Imprinted Polymer in Aqueous Solution
}

\author{
Nazia Tarannum, Meenakshi Singh* \\ Department of Chemistry, Mahila Mahavidyalaya, Banaras Hindu University, Varanasi, India \\ E-mail: "meenakshi_s4@rediffmail.com \\ Received August 26, 2011; revised October 1, 2011; accepted October 8, 2011
}

\begin{abstract}
Molecularly imprinted polymers selective for L-aspartic acid (LAA) have been prepared using the carboxybetaine polymer bearing zwitterionic centres along the backbone. LAA is well known to promote good metabolism, treat fatigue and depression along with its significance in accurate age estimation in the field of forensic science and is an important constituent of 'aspartame', the low calorie sweetener. In order to study the intermolecular interactions in the prepolymerization mixture between the monomer and the template (LAA)/non-template (DAA), a computational approach was developed. It was based on the binding energy of the complex between the template and functional monomer. The results demonstrate that electrostatic interactions primarily guide the imprinting protocol. The MIP was able to selectively and specifically take up LAA from aqueous solution, human blood serum and certain pharmaceutical samples quantitatively. Hence, a facile, specific and selective technique to detect the amino acid, LAA in the presence of various interferrants, in different kinds of matrices is presented.
\end{abstract}

Keywords: Amino Acid, L-Aspartic Acid, Molecularly Imprinted Polymer (MIP), Computational Modeling, Carboxybetaine Polymer, Selective Recognition

\section{Introduction}

L-Aspartic acid promotes robust metabolism and is occasionally used to treat fatigue and depression. The citric acid cycle, in which other amino acids and biochemicals (for example aspargine, arginine, lysine, methionine, threonine and isoleucine) are synthesized, requires aspartic acid as an important intermediary [1]. Moreover, aspartic acid treats chronic fatigue by playing a significant role in generating cellular energy. It moves the coenzyme NADH molecules from the main body of the cell to its mitochondria to facilitate ATP synthesis. It removes excess toxins from the cells, particularly ammonia, which is detrimental to the brain, nervous system and liver also. It also assists transportation of minerals to form healthy RNA and DNA to the cells, and support the immune system by promoting increased production of immunoglobulins and antibodies (immune system proteins). It keeps our mind sharp by increasing NADH level in the brain, which boosts the production of neurotransmitters and chemicals needed for healthy mental state. Aspartic acid is found in dairy, beef, poultry, sugarcane and molasses. People with diets low in protein or with eating disorders or malnutrition may develop a deficiency, not only in aspartic acid but in other amino acids as well, and experience fatigue or depression. Athletes may need to take aspartic acid which can be found in protein supplements such as amino acid tablets and whey protein drinks/bars, and are often marketed as energy boosters. Furthermore, the world market for this amino acid is ever increasing since its direct application as a raw material for "Aspartame", a low calorie sweetener. Additionally, the analysis of aspartic acid racemization of dentine protein is used for accurate estimation of age in forensic science [2]. Such vital estimations with their importance in neurotransmission and other significant physiological activities and related dysfunctions warrant the development of an efficient, accurate, precise and selective tool for the isolation, identification and determination of aspartic acid from biological fluids as well as from other pharmaceutical samples.

The polymerization of monomers in the presence of a target molecule that imprints structural information into resulting network polymers, called molecularly imprinted polymer (MIP) is a scientific field that is rapidly gaining significance for a wide range of applications in 
chemistry, biotechnology and pharmaceutical research. MIPs are stable to physical and chemical treatment, including high temperature, pressure, extreme $\mathrm{pH}$, organic solvents, acids and bases [3]. In addition, MIPs can be reused many times by repeating extraction with suitable solvents and without remarkable decrease in the adsorption capacity for template molecules [4]. These properties have made them extremely attractive for solving problems in the fields of preparative chemical separations, chemical sensing or selective catalysis. Due to their analytically useful properties, such as selectivity, shelf stability, robustness and reusability, MIP offers potential for the synthesis of artificial recognition material and they are being proposed for the development of novel biorecognition techniques for human health and bioterrorism protection technologies.

MIP for aspartic acid was synthesized by the Mosbach group as a part of novel utilization of MIPs in the enzymatic synthesis of "aspartame" and Syritski et al. $[5,6]$ have also successfully imprinted aspartic acid on the electrochemically synthesized film of polypyrrole but many more aspects affecting the imprinting protocol need to be developed and endorsed. Herein we attempted the imprinting of LAA with a different approach, keeping in mind their native nature of zwitterionic composition, the polymer format chosen for imprinting is also a novel zwitterionic polymer synthesized in our laboratory [7] to enable the electrostatic interactions to direct in the template- monomer interactions responsible for selective and specific imprinting. In conventional molecular imprinting, a high level of cross-linking is used to ensure robustness. Template binding specificity and the slow rebinding kinetics arising from the inner diffusion of target molecules toward the recognition sites which are totally embedded in the polymer matrices hinder and slow down the overall uptake of the template. To prevail over this limitation, here in this novel polymer format external crosslinker is not needed as in this class of carboxybetaine polymers or zwitterionic polymers in general, the electrostatic interactions between the two charge centres keep them in collapsed state, which can be considered as self crosslinking forces. Low mol wt electrolytes or other stimuli are needed to break these coulombic interactions. The biocompatible and biomimetic nature of the carboxybetaines has recently been applied for wide range of biomedical applications [8-11].

A typical imprinting protocol is tedious, time consuming and labour-intensive. An attempt was made to simulate the adduct or complex formed between the monomer/template (LAA) and the monomer/non-template (DAA), the enantiomer of LAA. For assessment of the template and monomer affinities, binding energy $(\Delta \mathrm{E})$ of the template and monomer can be used as a measure of their interaction [12-16]. Additionally Mulliken charges on atoms and dipole moment has also been analysed to see the effect on imprinting.

\section{Materials and Methods}

\subsection{Reagents}

p-Phenylene diamine, glutaraldehyde, dimethylformamide (DMF), $\gamma$-butyro lactone, L-aspartic acid (LAA) and D-aspartic acid (DAA) were purchased from Loba Chemie (Mumbai, India). Aspartame was obtained from Sigma Aldrich (Steinheim, Germany). The interferrants studied like urea, oxalic acid, itaconic acid, malonic acid, L-glutamic acid and 4-aminobutyric acid were purchased from Spectrochem Pvt. Ltd. (Mumbai, India). Butanol, acetic acid and ninhydrin used for paper chromatography were procured from Loba Chemie (Mumbai, India). All the chemicals were of AR grade and used as received. Water used was triple distilled deionozed water $(0.05-$ $0.08) \times 10^{-6} \mathrm{~S} / \mathrm{cm}$. Human blood serum was collected from a local pathology centre and used as received. The pharmaceutical samples analyzed were Sugar free gold sachet (artificial sweetener) from Acme diet care Pvt. Ltd. (Chhatral, India). Sugar free gold was pretreated before use.

\subsection{Equipments}

A Cary 50 Bio (Varian Instrument Inc., Melbourne, Australia) was used for all spectrophotometric measurements. The infrared spectra of the materials were recorded using Jasco FTIR 5300 from $400-4000 \mathrm{~cm}^{-1}$. pH of the solutions were determined by Systronics $\mu \mathrm{pH}$ system 361 . Scanning electron microscopy was performed by FESEM Quanta200F.

\subsection{Computational Method}

All computer simulations were undertaken by the software Gaussian software [17]. The 3D chemical structures were generated using Gaussian View [18]. Further, geometry optimization and energy calculations were performed using DFT method. The DFT calculation is commonly applied to MIP studies as it has the advantages of high accuracy level of information, reliability and reasonable computational costs in comparison with other computational methods (e.g. ab initio) [19]. Therefore, DFT method was selected to set up the calculations. The geometry optimizations were performed at B3LYP/ 6-31G level of theory. Since the molecules under consideration are large in size and charged molecules, a larger basis set was adopted and the molecules were opti- 
mized again with the method [B3LYP/6-311G $(d, p)]$. To obtain better interaction energies, additional single point calculations were performed at the optimized geometries using the method [B3LYP/6-311G $(\mathrm{d}, \mathrm{p})]$.

\subsection{Preparation of Molecularly Imprinted Polymers}

Moleculary imprinted polymer and corresponding nonimprinted polymer poly (N-phenylene N'imino pentyl) imminium butane carboxylate were prepared and characterized as reported earlier [7]. In short, equimolar solutions of p-phenylene diamine were added to a solution of glutaraldehyde in DMF and the mixture was heated at 45 ${ }^{\circ} \mathrm{C}$ for three hours. The reaction mixture was subsequently treated with $\gamma$-butyrolactone. Before the gelation starts, $3.5 \mathrm{ml}$ of aqueous solution of LAA [1.082 g/L, pH $=2.80]$ was added. Gelation started after 20 minutes and imprinted adduct was obtained. It is to mention here that the polymer is not a hydrogel but an organogel. The corresponding structures of MIP and adduct are shown in Scheme 1.

The concentrations of LAA in the solutions were determined using a spectrophotometer at $200 \mathrm{~nm}$. Standard calibration plot was constructed at $200 \mathrm{~nm}$ and various parameters (e.g. extracting solvent, time, $\mathrm{pH}$ ) were optimized at $200 \mathrm{~nm}$. Deionized water at $40^{\circ} \mathrm{C}$ was used to extract LAA template from adduct (volume $10 \mathrm{~mL}$, temperature $40^{\circ} \mathrm{C}, \mathrm{n}=4$, time $20 \mathrm{~min}$ ). A weighed amount of imprinted polymer was dipped in the template solution under optimized conditions and subsequently washed with deionized water $\left(40^{\circ} \mathrm{C}\right)$. Further, the concentrations of solutions were calculated using standard calibration plot of LAA. All the measurements were made in triplicate (RSD $1.2 \%)$.

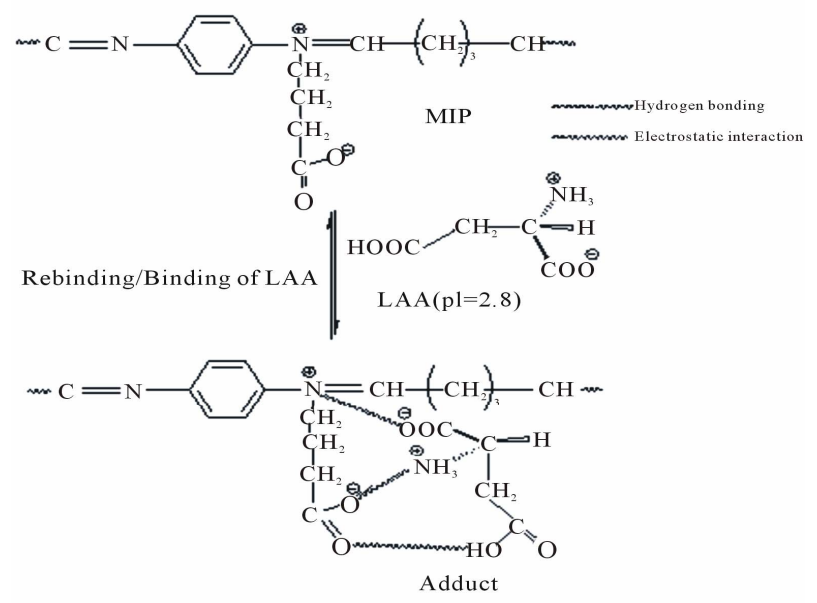

Scheme 1. Schematic route showing binding/rebinding of print molecule (LAA) in the polymer matrix.

\section{Results and Discussion}

Aspartic acid bears a net negative charge at neutral $\mathrm{pH}$ and no charge at $\mathrm{pI}(2.8)$ as the zwitterionic character of the amino acids cancel out the positive charge of $-\mathrm{NH}_{3}^{+}$ and negative charge of $-\mathrm{COO}^{-}$moiety at their respective pI. Thus we chose a zwitterionic polymer to imprint aspartic acid, which could provide electrostatic interactions with respective counter charges along with additional H-bonding and other hydrophobic interactions to generate specific recognition sites in the imprinting network. The biocompatibility of this polymer format chosen for imprinting LAA would be an added advantage for its utilization in developing the biorecognition techniques and applying the tool to biological fluids and other such pharmaceutical samples without any kind of pre-treatment with their application in forensic sciences also.

\subsection{Calculation of Interaction Energy}

The recognition ability of MIP is generally evaluated by affinity for template. The factors affecting affinity of the template for MIP are strength and quantity of interactions between monomers in polymer network and the template. The optimized geometries of non-imprinted polymer, imprinted polymer with template L-aspartic acid and complex with non-template D-aspartic acid are shown in Figure 1. In designing MIP via computational approach, monomers giving highest $\Delta \mathrm{E}$ with the template have been assumed to offer corresponding MIP with highest affinity for the chosen template. The interaction energies of the template with complex were calculated from Equation (1):

$$
\Delta E=\left|E_{\text {complex }}-E_{\text {template }}-\sum E_{\text {monomer }}\right|
$$

where $E_{\text {complex }}$ is the energy of the complex, $E_{\text {template }}$ is the energy of the template and $\mathrm{E}_{\text {monomer }}$ is energy of the monomer unit.

The results are shown in Tables 1-3. Tables 1-2 show the Mulliken atomic charges in complexes L and D. For the sake of brevity only those atoms involved in interactions responsible for imprinting such as electrostatic interactions, H-bonding and other weak forces are shown in the tables. Figure 2 shows the electrostatic interactions and $\mathrm{H}$-bonding interactions between template and monomer. As the dipole moments of complex $\mathrm{L}$ as well as $\mathrm{D}$ are much larger, the electrostatic interactions seem to play a primary role in the interaction of template with MIP cavities.

A comparison of Mulliken charges before imprinting and after imprinting on the atoms involved either in electrostatic interaction or in H-bonding suggests a more stable structure [20]. Hence, imprinting the polymer with 


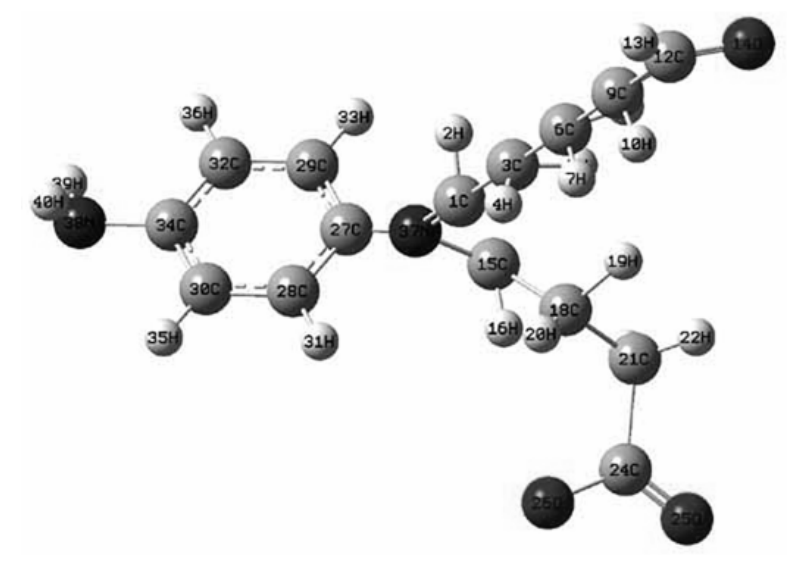

(a)

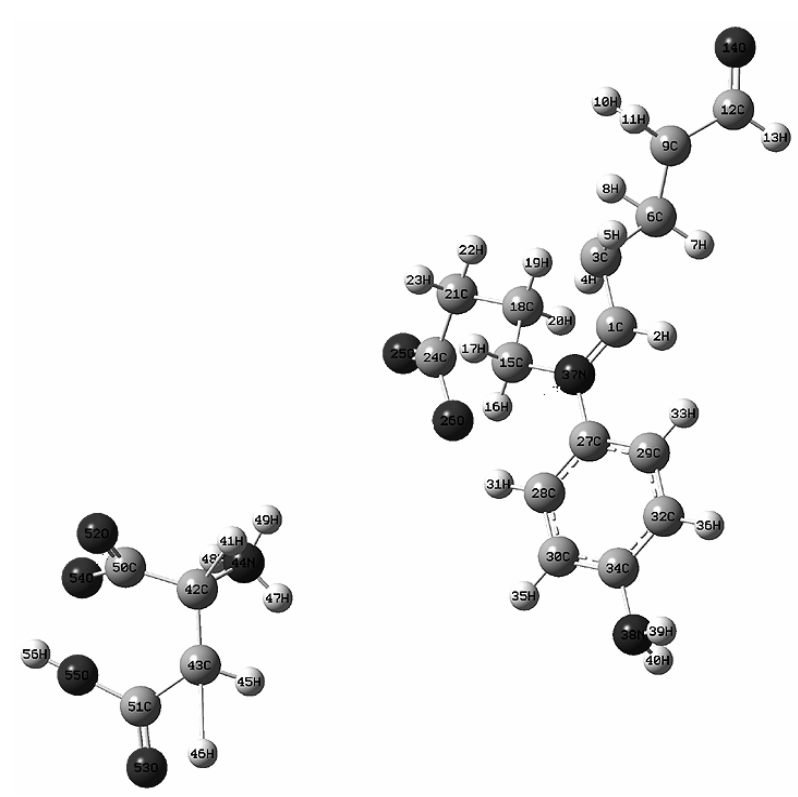

(b)

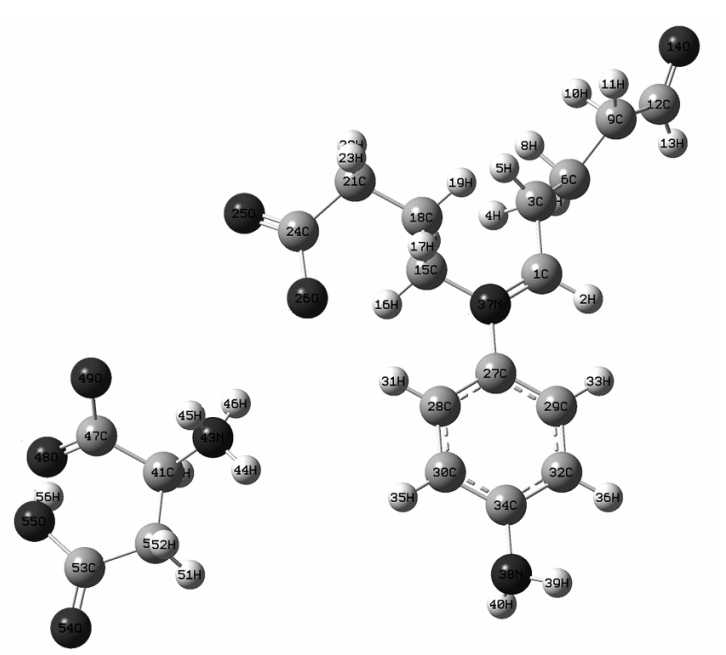

(c)

Figure 1. Optimized structures of (a) NIP, (b) Complex L and (c) Complex D.
Table 1. Mulliken charges on the atoms of Complex $L$.

\begin{tabular}{ccc}
\hline Atom & Atom Number & Mulliken atomic charge \\
\hline $\mathbf{C}$ & 1 & 0.121 \\
$\mathbf{H}$ & 2 & 0.137 \\
$\mathbf{C}$ & 15 & -0.058 \\
$\mathbf{H}$ & 16 & 0.225 \\
$\mathbf{H}$ & 17 & 0.122 \\
$\mathbf{O}$ & 25 & -0.538 \\
$\mathbf{O}$ & 26 & -0.680 \\
$\mathbf{C}$ & 27 & 0.290 \\
$\mathbf{N}$ & 37 & -0.460 \\
$\mathbf{N}$ & 43 & -0.481 \\
$\mathbf{H}$ & 44 & 0.320 \\
$\mathbf{H}$ & 45 & 0.327 \\
$\mathbf{H}$ & 46 & 0.368 \\
$\mathbf{O}$ & 49 & -0.624 \\
$\mathbf{H}$ & 56 & 0.275 \\
\hline & & \\
\hline & &
\end{tabular}

Table 2. Mulliken charges on the atoms of Complex D.

\begin{tabular}{ccc}
\hline Atom & Atom Number & Mulliken atomic charge \\
$\mathbf{C}$ & 1 & 0.136 \\
$\mathbf{H}$ & 2 & 0.176 \\
$\mathbf{C}$ & 15 & -0.086 \\
$\mathbf{H}$ & 16 & 0.283 \\
$\mathbf{H}$ & 17 & 0.180 \\
$\mathbf{O}$ & 25 & -0.481 \\
$\mathbf{O}$ & 26 & -0.612 \\
$\mathbf{C}$ & 27 & 0.299 \\
$\mathbf{N}$ & 37 & -0.586 \\
$\mathbf{N}$ & 44 & -0.696 \\
$\mathbf{H}$ & 47 & 0.362 \\
$\mathbf{H}$ & 48 & 0.387 \\
$\mathbf{H}$ & 49 & 0.405 \\
$\mathbf{O}$ & 54 & -0.606 \\
$\mathbf{H}$ & 56 & 0.468 \\
\hline
\end{tabular}

the template LAA could be proceeded experimentally.

As shown in Table 3, dipole moments of the nonimprinted polymer, L-aspartic acid imprinted polymer (complex L) and complex D with non-template possess 


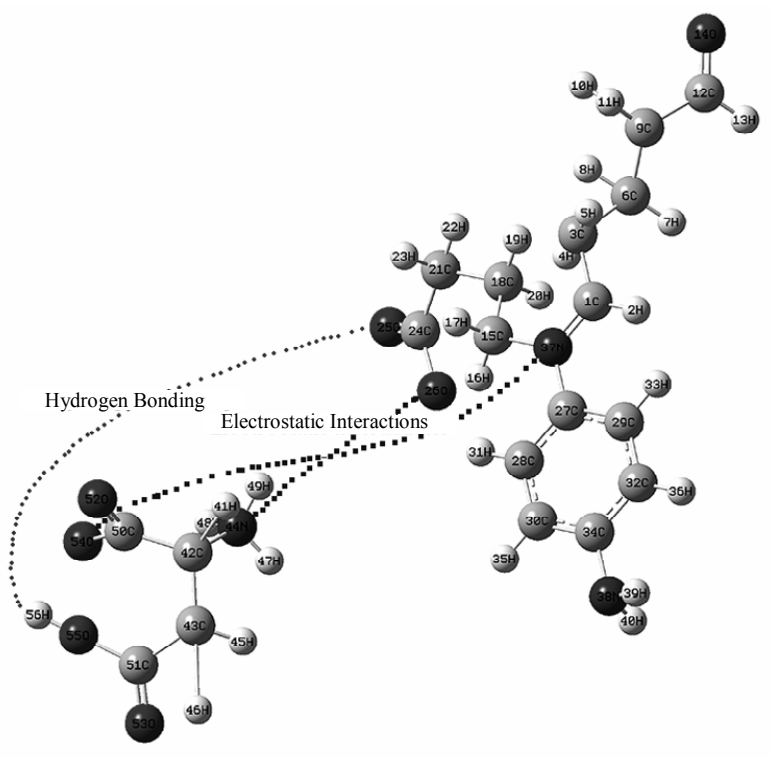

(a)

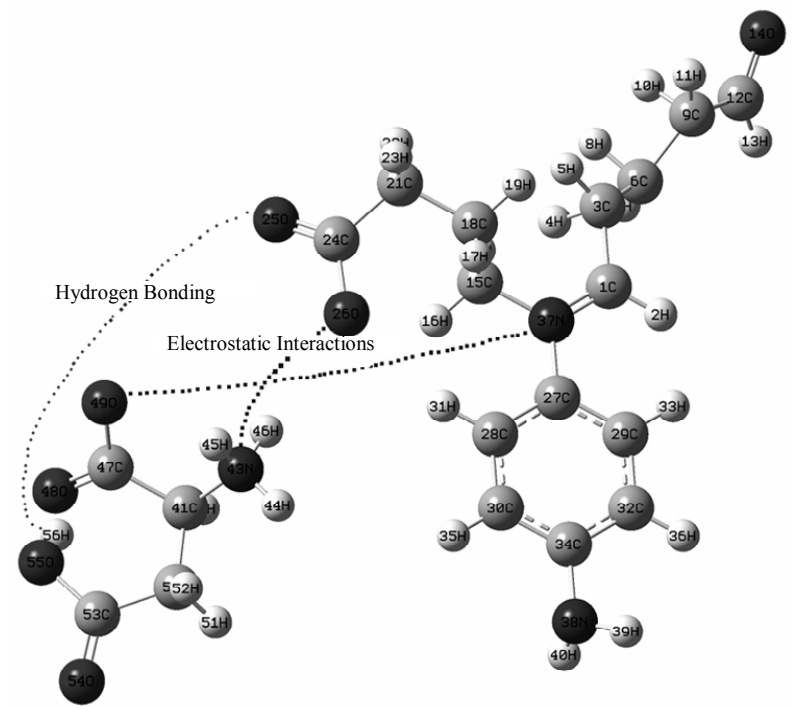

(b)

Figure 2. Interactions between monomer and template in (a) complex $L$ and non-template in (b) complex $D$.

quite high dipole moment. Dipole moment is a measure of polarity of the molecule in general and polarity of the bond in particular. When molecules (here template and monomers) approach each other, the initial contact arises from long-range electrostatic forces and when they approach each other, these electrostatic forces are supplemented by the weak forces (H-bonding, Vander Waal forces, hydrophobic interactions and $\pi$ - $\pi$ interactions) between complementary functional groups located on template molecule and on the monomers thus fitting the template into thus created specific MIP cavities. The $\Delta \mathrm{E}$ value of complex $L$ as shown in the Table 3 is higher than that of complex D. Thus the LAA imprinted in the carboxybetaine polymeric network is more stable. Hence, the imprinting of LAA was conceded further.

\subsection{Infrared Spectrum}

In MIP technology, FTIR analysis is useful for preliminary determination of template-MIP interaction which proves to be vital for prediction of stability and interaction capacity of recognition cavities towards template molecule and detection of the efficacy extraction and rebinding procedures. The FTIR spectra of adduct and MIP has been shown in Figure 3.

In the non-imprinted polymer (NIP), the $-\mathrm{NH}_{2}$ group from phenylene diamine end groups and $-\mathrm{OH}$ stretch (moisture) conferred an overlapped absorption band at -3430 . The C-H asymm st at $2925 \mathrm{~cm}^{-1}$ and C-H symm st at $2859 \mathrm{~cm}^{-1}$ characterize the $-\mathrm{CH}_{2}$ groups of the skeleton. The carboxylate group $\left(\mathrm{COO}^{-}\right)$is well evinced by characteristic absorption bands at $1167 \mathrm{~cm}^{-1}$ (C-CO-C) with shoulders at 1093 and $1035 \mathrm{~cm}^{-1}$. In the adduct, the characteristic $-\mathrm{NH}_{3}^{+}$st of LAA is observed at 3370 $\mathrm{cm}^{-1}$ and $3399 \mathrm{~cm}^{-1}$ as a part of overlapped broad absorption band. Since the $-\mathrm{NH}_{3}^{+}$groups are shielded by electrostatic binding with the $\mathrm{COO}^{-}$group of carboxybetaine group of polymer, the characteristic $\mathrm{COO}^{-}$absorptions at 1173, 1120 and 1083 are subdued in intensity. Since neither of the carboxyl groups are free (from polycarboxybetaine or from LAA, the intensity of the peaks are diminished to a larger extent. But on extracting the LAA from adduct, in the resulting MIP (Figure 3), the characteristic carboxylate absorption bands regained their intensity at $1175 \mathrm{~cm}^{-1}, 1100 \mathrm{~cm}^{-1}$ and $1061 \mathrm{~cm}^{-1}$ attesting the removal of LAA which shrouded the charged centres of polycarboxybetaine. Since imine centres are devoid of any covalently bonded $\mathrm{H}$, similar analogy on the charged centres of imine could not be applied in absence of N-H bond. The other characteristic absorption band of the polymeric chain shifts from 2859 (C-H sym st) to $2890 \mathrm{~cm}^{-1}$ in adduct and again to 2835 $\mathrm{cm}^{-1}$ in MIP. This shift might be attributed to a more ordered orientation of functional groups with the structural backbone also. Whereas in NIP, the structural backbone was free to orient in absence of any template only according to its own functional groups but in MIP and adduct, the presence of template dictates the spatial orientation of groups, hence a little change is observed in $\mathrm{C}-\mathrm{H}$ st absorptions. The (C-CO-C) absorption of carboxyl have lost its sharpness in adduct evincing interaction of template LAA with MIP via electrostatic interaction of carboxyl groups of carboxybetaine polymer. The absorption bands at $1167,1093,1035 \mathrm{~cm}^{-1}$, with 1767 $\mathrm{cm}^{-1}$, the typical absorptions of carboxylate entity, has lost their sharpness as they are involved in electrostatic interactions with template as shown in Scheme 1. 
Table 3. The interaction energies and dipole moments of monomer constituents and complexes between monomer and template (LAA)/non-template (DAA).

\begin{tabular}{|c|c|c|c|c|}
\hline S. No. & Compounds & Energy (Hartree) in gas phase DFT B3LYP/6311G(d,p) & Interaction Energy $(\Delta \mathrm{E})$ in $\mathrm{KJ} / \mathrm{mol}$ & $\begin{array}{l}\text { Dipole moment } \\
\text { (Debye) }\end{array}$ \\
\hline 1. & $\begin{array}{l}\text { Glutaraldehyde } \\
\text { (monomer) }\end{array}$ & -345.85 & - & 2.957 \\
\hline 2. & $\begin{array}{l}\text { p-Phenylene diamine } \\
\text { (monomer) }\end{array}$ & -342.04 & - & 0.001 \\
\hline 3. & $\gamma$-butyrolactone & -306.58 & - & 4.548 \\
\hline 4. & Nonimprinted polymer & -918.99 & - & 16.313 \\
\hline 5. & Complex L & -1431.16 & 11468 & 24.571 \\
\hline 6. & Complex D & -1431.45 & 11425 & 23.210 \\
\hline 7. & LAA(template) & -512.47 & - & 3.126 \\
\hline 8. & DAA (non-template) & -512.46 & - & 1.093 \\
\hline
\end{tabular}

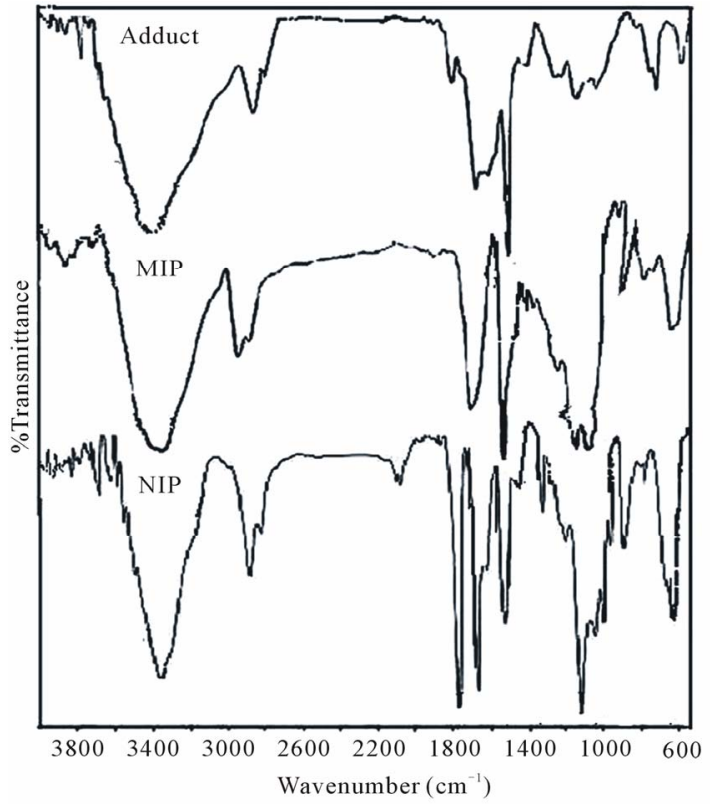

Figure 3. Comparative FTIR of adduct and molecularly imprinted polymer (MIP).

Electrostatic interaction has been known to play a primary guiding role in inducing the other kinds of non-covalent weak interactions to specifically and selectively create the imprinted cavities for the amino acid as the template has been reported by earlier workers also [21]. The adduct shows $\mathrm{NH}_{2}$ wagging at $797 \mathrm{~cm}^{-1}$. The other absorption bands were characteristic of polymer network as reported earlier [7]. The fingerprint region clearly distinguishes MIP and adduct as shown in Figure 3.

\subsection{Scanning Electron Microscopy}

The scanning electron microscopy images of the NIP, adduct and MIP are shown in Figure 4. The images of adduct and the MIP are shown in Figures 4(b) and (c). Figure 4(b) shows some compactness which after template removal turned microporous and appeared to be consisted of cavities reflecting a highly porous material with microvoids. While the nonimprinted polymer surface appeared to have smooth surface and few pore structures as shown in Figure 4(a). Here the polymerization was carried out in the absence of template. The morphological changes are well-consistent with the imprinting results as discussed in subsequent section.

\subsection{Optimization of Analytical Parameters}

To find out the conditions under which binding of LAA to the LAA-imprinted polymer is best and much higher than binding of the NIP; the extracting solvent, its volume, extraction time and $\mathrm{pH}$ of the solution were optimized. Among the solvents chosen (water, ethanol, ethanol-water, chloroform, acetonitrile, etc.) for extracting the template molecule from MIP-template adducts, deionized water at $40^{\circ} \mathrm{C}$ was found to be best suited. The optimized extraction conditions are $10 \mathrm{ml}$ deionized water at $40^{\circ} \mathrm{C}$. Three washes of optimized extracting solvent with continuous mechanical stirring $(600 \mathrm{rpm})$ for 20 min were able to extract template completely. The presence of LAA in final wash was checked by paper chromatography $\left(\mathrm{CHCl}_{3}(1): \mathrm{CH}_{3} \mathrm{OH}(2)\right)$ using ninhydrin as developing reagent. In rebinding experiments, as shown in Figure 5, 40 min were optimum for uptake of the template in MIP cavities.

Figure 6 shows the concentration dependence of LAA imprinted polymer. The uptake by MIP was linearly dependent on concentration of the template with saturation of $1666 \mathrm{ppm}$ by $1 \mathrm{mg}$ of MIP, being attained at $0.02 \mathrm{M}$ of template LAA in aqueous solution.

As shown in Figure 7, pH range 2.7 - 6.0 was found to 


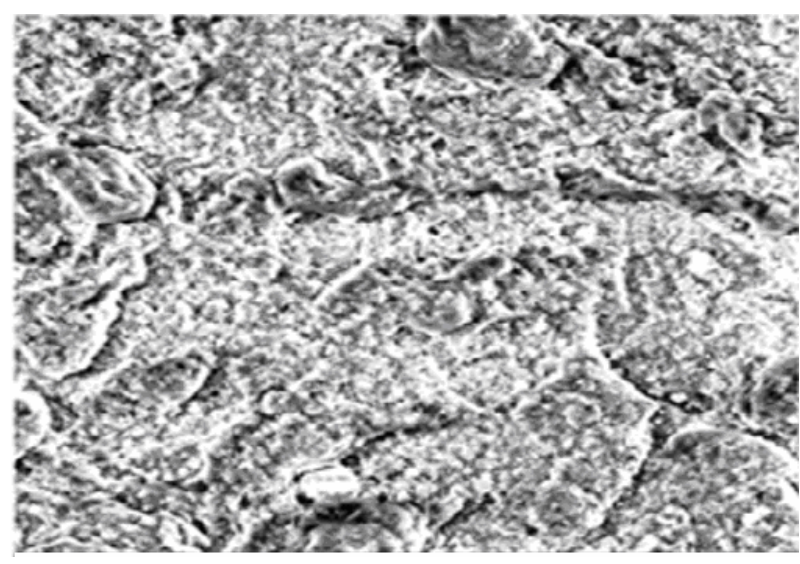

(a)

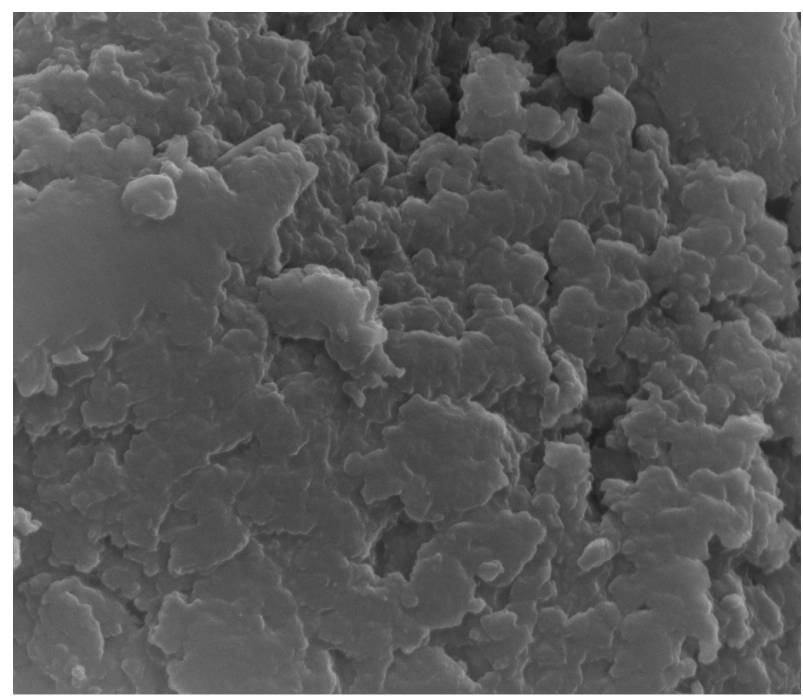

(b)

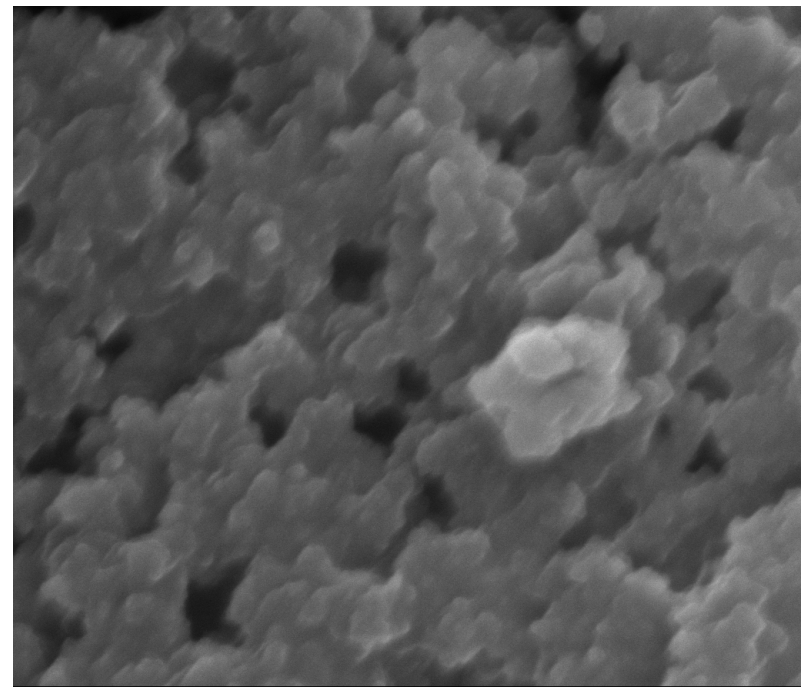

(c)

Figure 4. Scanning electron microscopy of (a) NIP (b) adduct and (c) MIP at $10000 \times$ magnification.

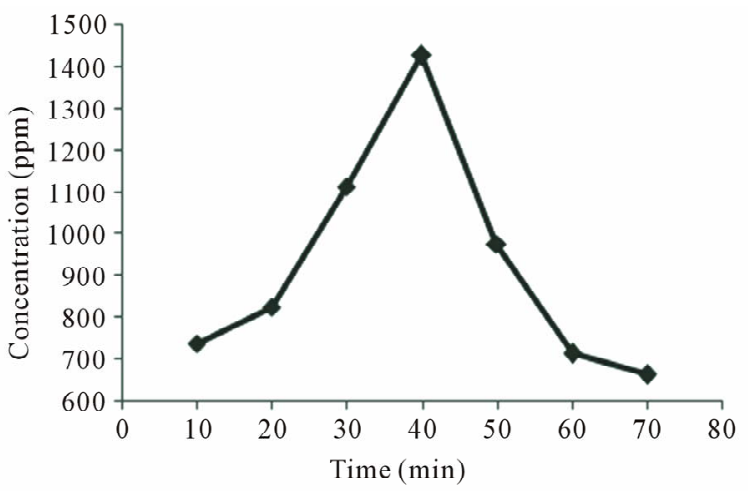

Figure 5: Effect of the retention time to rebind the template on molecularly imprinted polymer (MIP).

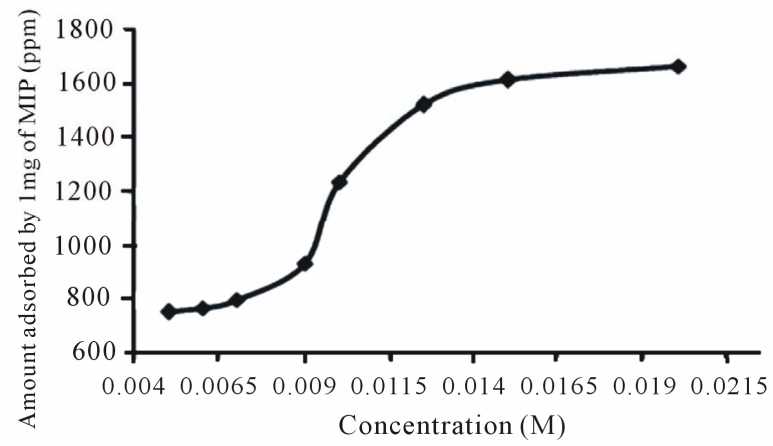

Figure 6. Effect of the concentration of template solution on recovery $(\%)$ of the template from aqueous solution.

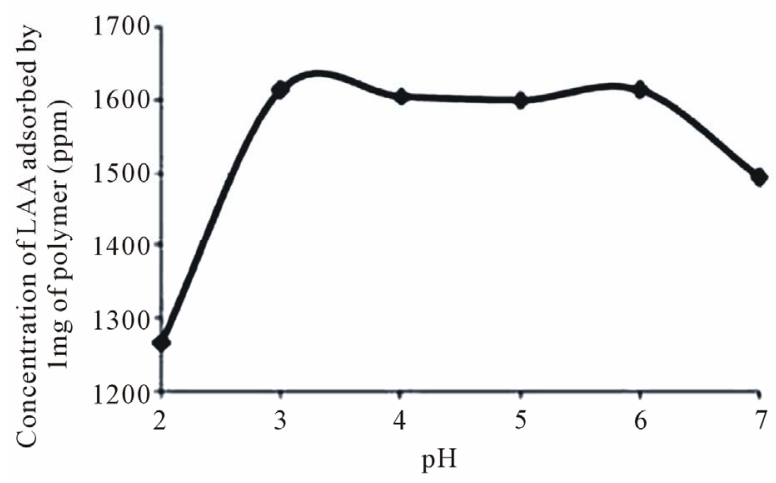

Figure 7. Effect of pH of the template solution on recovery (\%).

be most suitable for recovery of template. Scheme 2 shows the $\mathrm{pH}$ dependent ionization behaviour of aspartic acid. Below $\mathrm{pK}_{\mathrm{a} 1},(\mathrm{pH}<1.88)$ the amino acid LAA is fully protonated with net charge on the template as $(+1)$ in aqueous solutions. As $\mathrm{pH}$ is increased beyond $\mathrm{pK}_{\mathrm{a} 1}$, the net charge is 0 at its pI 2.88, when amino acids are in their zwitterionic form. The native nature of the polymer, i.e. zwitterionic nature which has a positive and a negative charge centres by virtue of the strength of their ionisable groups is accountable for this behaviour where the 
electrostatic interactions between the charge centres of imprinted polymer and the corresponding opposite charge centres of template LAA as shown in Scheme 2, dictate/dominate the retention mechanism. In the $\mathrm{pH}$ range $1.88-2.88$, the electrostatic interactions are accountable for tight binding of the amino acid to its MIP cavity (Scheme 2). In addition, H-bonding facilitates the imprinting further. As the $\mathrm{pH}$ of the solution increases further, in the range $2.8-6.0$, aspartic acid is deprotonated further with net charge as $(-1)$, electrostatic forces again keeps the template and hold them in the cavities found in MIP. As the Scheme 2 shows, the additional ionic interaction at $\mathrm{pH}>6.0$, further tightens the binding, hence making the imprinting more successful. Hence $\mathrm{pH}$ range $2.8-6.0$ was experimentally found to be suitable for this electrostatic force driven assembly of template-polymer assembly in this MIP generated.

Figure 8 shows the binding capacity in terms of Recovery (\%) of MIP and NIP for template LAA. The known weight of MIP and NIP samples were equilibrated under optimized conditions in aqueous LAA solutions of various concentrations. The MIP and NIP were washed and extracted with optimized solvents under the similar experimental conditions. MIP is envisioned as a selective binding material with functional groups complementary to the template structural features. The template fits into the imprinted cavity driven by the electrostatic interaction in this study followed by aligning of the functional groups on the MIP around the LAA conformational orientation. In NIP, although the functional groups are also present in the polymer, they are randomly arranged in such a manner that it is ineffective for correct binding with the template. Similarly, an interferrant is
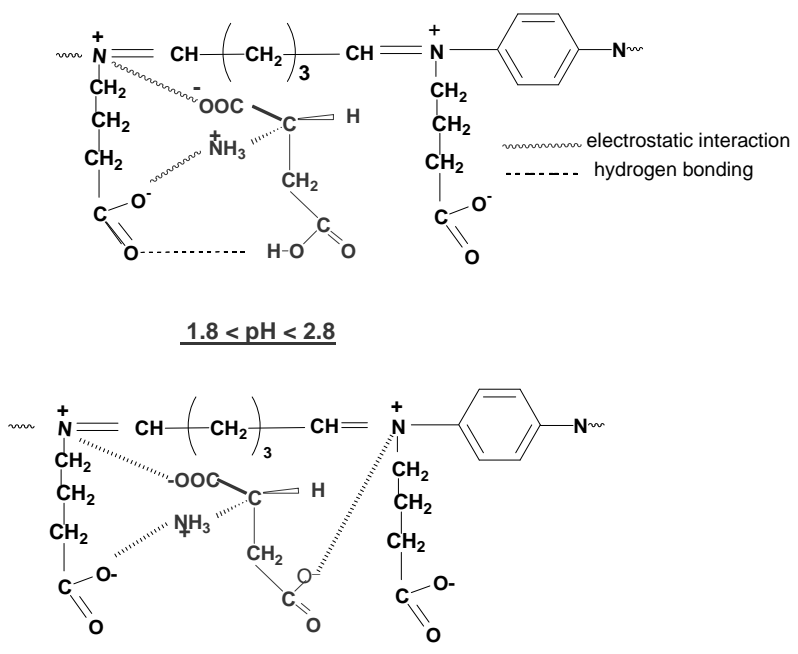

$2.8<\mathrm{pH}<6.0$

Scheme 2. Scheme representing the structure of adduct at varying $\mathbf{p H}$.

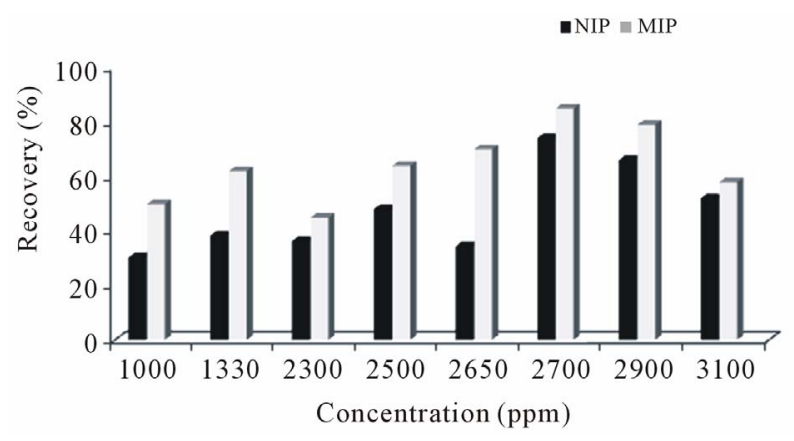

Figure 8. Comparison of selective recognition of analyte by NIP and MIP at different concentrations of template.

different in size and conformation and might lack key structural features responsible for fitting into the recognition cavities. In MIPs, the cavities created after removal of the template are complementary to the imprint molecule in size and coordination geometries. This leads to much greater affinity for the template molecule by molecular imprinting, in comparison with non-imprinted one. As the figure shows, MIP shows an imprinting effect for the template molecule. The non-specific bindings are responsible for the template adsorption shown by the NIP as evident in the figure. At lower concentration, the non-specific bindings are not able to override the specific bindings but as the crowding begins at high concentrations of template in solution, the adherence of template to the non-imprinted polymer network could be noted as the competition for the specific sites in the imprinted network becomes high.

In order to evaluate the selectivity of the synthesized MIP, various structural analogues of L-aspartic acid were considered (Figure 9 structure of interferrants).

The analogues chosen were either having similar functional groups or its isomer D-asapartic acid (DAA). Figure 10 shows the response of synthesized MIP toward template and other non-templates. From Figure 10, it is evident that the MIP is selective to LAA only even in presence of DAA or other possible interferrants having structural similarity. Hence, this study confirms the selectivity of MIP towards template.

The technique thus developed was validated by determining its performance characteristics regarding linearity, repeatability and precision. To test the UV response linearity, a series of standard solutions in the concentration range $0.005-0.015 \mathrm{M}$ was analyzed (at least 15 samples).

\section{Analytical Applications}

This MIP technique was also examined in human blood serum samples and in certain pharmaceutical samples. The serum collected from local pathology laboratory was 
<smiles>C=C(CC(=O)O)C(=O)OCC(=O)CC(=O)O</smiles>

Itaconic Acid<smiles>[NH3+][C@@H](CC(=O)O)C(=O)O</smiles>

D Aspartic Acid<smiles>NC(=O)[Mg]</smiles>

$$
\mathrm{CH}_{2}\left\langle\begin{array}{l}
\mathrm{COOH} \\
\mathrm{COOH}
\end{array}\right.
$$

Malonic Acid<smiles>[12NH2]C(=O)C(=O)O</smiles>

L Glutamic Acid

$$
4 \text { Aminobutyric Acid }
$$

Figure 9. Structures of analogues of LAA used in the cross-selectivity study of MIP.

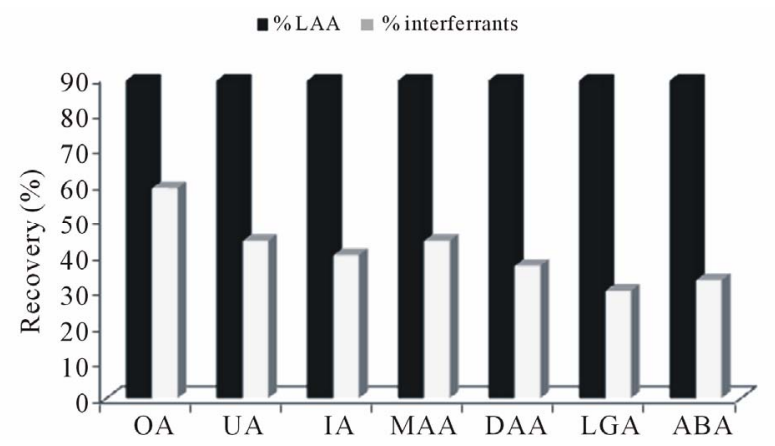

Figure 10. Cross-selectivity study of adsorption of various interferrants on LAA-MIP (OA-oxalic acid, UA-urea, IAitaconic acid, MA- malonic acid, DAA-D-aspartic acid, LGA-L-glutamic acid, ABA-4 aminobutyric acid).

diluted 50 times to minimize non-specific bindings due to intricate matrices of blood serum. Human blood serum is reported to contain $50 \mathrm{ppm}$ of L-aspartic acid. The MIP was immersed in dilute serum solution under optimized conditions. The extracted solution was measured to contain $40 \mathrm{ppm}$ LAA. In this way, the MIP thus developed was able to recover $80 \%$ of LAA present in human blood serum. This recovery can be enhanced further if such MIP could be hyphenated to other suitable extraction (Solid phase extraction or Solid phase microextraction) techniques. Further the serum sample was scanned spectrophotometrically before and after treat- ment with MIP. The key peaks responsible for LAA were found to be vanished after coming in contact with MIP under optimized conditions.

The pharmaceutical sample (Sugarfree gold) is reported to contain $35 \mathrm{mg}$ of aspartame, a dipeptide comprising of L-aspartic acid and methyl ester of L-phenylalanine. Aspartame in the pharmaceutical sample was hydrolyzed by acid $(6 \mathrm{M} \mathrm{HCl})$ to release the constituent amino acids. The hydrolytic products were verified by paper chromatography [butanol $(25 \mathrm{~mL})$ : acetic acid $(5$ $\mathrm{mL})$ : water $(20 \mathrm{~mL})$. The total LAA recovered from the sample was $75 \%$.

\section{Conclusions}

The described LAA-MIP has shown enantioselective feature for the trace-level analysis of d- and 1-LAA. For this a shape complementry cavity in the imprinted polymeric network was created. The binding mechanism involved principally electrostatic interactions complemented with hydrogen bonding between the analyte and MIP, which reduced non-specific bindings of structural analogues and potential interferents. The developed MIP can be regenerated for next uses maintaining enantioselective separation. Hence, a facile, highly specific and selective technique to detect the amino acid LAA in the presence of various interferrants, in different kinds of matrices and most importantly without any pre-treatment or other such complicated pathological/clinical procedures is presented here.

\section{Acknowledgements}

The authors are grateful to Dr. R. K. Singh for his kind cooperation extended in Gaussian calculations. Financial grant was provided by Department of Science and Technology, New Delhi (SR/S2/CMP-65/2007).

\section{References}

[1] M. Di. Pasquale, "Amino Acids and Proteins for the Athlete the Anabolic Edge," CRC Press, Boca Raton, 1997.

[2] E. R. Waite, M. J. Collins, S. Ritz-Timme, H.-W. Schutz, C. Cattaneo and H. I. M. Borrman, "A Review of the Methodological Aspects of Aspartic Acid Racemisation analysis for Use in Forensic Science," Forensic Science International, Vol. 103, No. 2, 1999, pp. 113-124. doi:S0379-0738(99)00081-X

[3] P. A. G. Cormack and A. Z. Elorza, "Molecularly Imprinted Polymers: Synthesis and Characterisation," Journal of Chromatography B, Vol. 804, No. 1, 2004, pp. 173-182. doi:10.1016/j.jchromb.2004.02.013

[4] G. Baydemir, N. Bereli, M. Andaç, R. Say, I. Y. Galaev and A. Denizli, "Bilirubin Recognition via Molecularly 
Imprinted Supermacroporous Cryogels," Colloids and Surfaces B: Biointerfaces, Vol. 68, No. 1, 2009, pp. 33-38. doi:10.1016/j.colsurfb.2008.09.08

[5] L. Ye, O. Ramström, M.-O. Månsson and K. Mosbach, "A New Application of Molecularly Imprinted Materials," Journal of Molecular Recognition, Vol. 11, No. 1-6, 1998, pp. 75-78.doi:0952-3499/98/010075-04

[6] V. Syritski, J. Reut, A. Menaker, R. E. Gyurcsànyi and A. Öpik, "Electrosynthesized Molecularly Imprinted Polypyrrole Films for Enantioselective Recognition of L-Aspartic Acid," Electrochimica Acta, Vol. 53, No. 6, 2008, pp. 2729-2736. doi:10.1016/j.electacta.2007.10.032

[7] N. Tarannum and M. Singh, "Synthesis and Characterization of Zwitterionic Organogels Based on Schiff Base Chemistry," Journal of Applied Polymer Science, Vol. 118, No. 5, 2010, pp. 2821-2832. doi:10.1002/app.32393

[8] C. R. Emmenegger, E. Brynda, T. Riedel, Z. Sedlakova M. Houska and A. B. Alles, "Interaction of Blood Plasma with Antifouling Surfaces," Langmuir, Vol. 25, No. 11, 2009, pp. 6328-6333. doi:10.1021/la900083s

[9] S. Fujishita, C. Inaba, S. Tada, H. Kitano, M. Gemmei-Ide and Y. Saruwatari, "Effect of Zwitterionic Polymers on Wound Healing," Biological \& Pharmaceutical Bulletin, Vol. 31, No. 12, 2008, pp. 2309-2315. doi:10.1248/bpb.31.2309

[10] W. Yang, L. Zhang, S. Wang, A. D. White and S. Jiang, "Functionalizable and Ultra Stable Nanoparticles Coated with Zwitterionic Poly(Carboxybetaine) in Undiluted Blood Serum," Biomaterials, Vol. 30, No. 29, 2009, pp. 5617-5621. doi:10.1016/j.biomaterials.2009.06.036

[11] W. Yang, H. Xue, W. Li, J. Zhang and S. Jiang, "Pursuing 'Zero' Protein Adsorption of Poly(Carboxybetaine) from Undiluted Blood Serum and Plasma," Langmuir, Vol. 25, No. 19, 2009, pp. 11911-11916. doi:10.1021/la9015788 11911

[12] F. Faridbod, M. R. Ganjali, R. Dinarvand, P. Norouzi and S. Riahi, "Schiff's Bases and Crown Ethers as Supramolecular Sensing Materials in the Construction of Potentiometric Membrane Sensors," Sensors, Vol. 8, No. 3, 2008, pp. 1645-1703. doi:10.3390/s8031645

[13] F. Faridbod, M. R. Ganjali, B. Larijani, P. Norouzi, S. Riahi and F. F. Mirnaghi, "Lanthanide Recognition: An asymetric Erbium Microsensor Based on a Hydrazone
Derivative," Sensors, Vol. 7, No. 12, 2007, pp. 3119-3135. doi:10.3390/s7123119

[14] M. R. Ganjali, P. Norouzi, F. Faridbod, S. Riahi, J. Ravanshad, J. Tashkhourian, M. Salavati-Niasari and M. Javaheri, "Determination of Vanadyl Ions by a New PVC Membrane Sensor Based on N,N'-Bis-(salicylidene)-2,2dimethylpropane-1,3-diamine," IEEE Sensors Journal, Vol. 7, No. 4, 2007, pp. 544-550. doi:10.1109/JSEN.2007.891986

[15] M. R. Ganjali, P. Norouzi, F. S. Mirnaghi, S. Riahi and F. Faridbod, "Lanthanide Recognition: Monitoring of Praseodymium (III) by a Novel Praseodymium (III) Microsensor Based on N-(Pyridin-2-ylmethylene) Benzohydrazide 1,3-diamine," IEEE Sensors Journal, Vol. 7, No. 8, 2007, pp. 1138-1144. doi:10.1109/JSEN.2007.897950

[16] S. Riahi, M. R. Ganjali, A. B. Moghaddam and P. Norouzi, "Experimental and Quantum Chemical Study on the IR, UV and Electrode Potential of 6-(2,3-Dihydro1,3-di-oxo-2-phenyl-1H-inden-2-yl)-2,3-dihydroxybenzal dehyde," Spectrochimica Acta Part A, Vol. 71, No. 4, 2008, pp. 1390-1396. doi:10.1016/j.saa.2008.04.016

[17] M. J. Frisch, G. W.Trucks, H. B. Schlegel, G. E. Scuseria, M. A. Robb, J. R. Cheeseman, et al., "Gaussian 03, Revision D.01," Gaussian, Inc., Wallingford, 2004.

[18] R. Dennington, T. Keith and J. Millam, "GaussView 4.1," Semichem, Inc., Shawnee Mission, 2007.

[19] S. Riahi, F. Edris-Tabrizi, M. Javanbakht, M. R. Ganjali and P. Norouzi, "A Computational Approach to Studying Monomer Selectivity towards the Template in an Imprinted Polymer," Journal of Molecular Modeling, Vol. 15 , No. 7, 2009, pp. 829-836. doi:10.1007/s00894-008-0437-2

[20] A. Kowalska, A. Stobiecka and S. Wysocki, "A Computational Investigation of the Interactions between Harmane and the Functional Monomers Commonly Used in Molecularly Imprinting," Journal of Molecular Structure: THEOCHEM, Vol. 901, No. 1-3, 2009, pp. 88-95. doi:10.1016/j.theochem.2009.01.008

[21] S. A. Piletsky, H. S. Andersson and I. A. Nicholls, "Combined Hydrophobic and Electrostatic InteractionBased Recognition in Molecularly Imprinted Polymers," Macromolecules, Vol. 32, No. 3, 1999, pp. 633-636. doi:10.1021/ma9812736 